\title{
Compassion and Compensation for Polio Cases
}

\section{Yash Paul*}

Consultant Pediatrician, Maharaja Agrasen Hospital, Vidhyadhar Nagar, Jaipur 302023, India

\begin{abstract}
Aim: To find if some children had developed paralytic poliomyelitis despite participating in the national polio eradication program.

Methods: Data of National Polio Surveillance Project regarding polio eradication program in India was analysed.

Results: Many children have developed paralytic poliomyelitis despite taking many doses of OPV because of vaccine failure and many children have developed VAPP after taking OPV and some children have developed VAPP because of secondary spread of mutant vaccine polio viruses.

Conclusion: The children who have developed polio during polio eradication program are considered as 'price to be paid' for larger interest of the society. Though the harm occurred to these children was not intentional but, nevertheless it was foreseeable. Thus, adequate compensation should be paid to such children.
\end{abstract}

Keywords: Polio eradication; Polio cases; Compassion; Compensation

\section{Introduction}

Global polio eradication program was launched in 1988, and now it appears that soon polio eradication will be achieved. The policy makers have to prepare post eradication strategy which may be same or different for different countries. There is a need for discussion at international level whether those children who have developed paralytic poliomyelitis during the global polio eradication program be considered 'a price to be paid' or treated with empathy and compassion for the unintentional but foreseeable harm which had occurred to these children. These children rightfully deserve suitable compensation for their life long physical handicap and suffering and not treated as a price paid by them for a noble cause. The author discusses the issue in the Indian perspective which may not be very different from the situations in other countries.

Last polio case from India was reported on January 13, 2011 from West Bengal, WHO has taken off India from the list of endemic areas. It is hoped that no more polio case will occur as efforts regarding polio eradication have not been slackened and in due course of time India may be declared polio free country. The Government of India should legislate an Act to provide compensation to those children who have developed polio during the national polio eradication campaign.

\section{Why Compensation?}

Global polio eradication was started in 1988. Oral polio vaccine (OPV) and inactivated polio vaccine (IPV) were available. Even at that time it was known that some children, especially from the developing countries may fail to show appropriate response to OPV, called vaccine failure and may develop paralytic polio even after taking appropriate number of OPV doses, and also that OPV can cause VAPP in vaccine recipients and close contacts of the vaccines. But, it was hoped that number of such cases would be low and polio eradication campaign will end by year 2000. Also the cost factor, oral route of administration, being a live vaccine it was supposed to provide long lasting immunity and quick onset of protection and above all, immunity induced in contacts by the secondary spread of live attenuated vaccine polioviruses were the reasons that OPV was chosen for global polio eradication.

During this long period of polio eradication campaign initiated in 1994 in Delhi, Rajasthan and Gujarat, extended on October 2, 1995 nation-wide some children have developed polio, treatment and rehabilitation services are being provided to these children. As these children have developed polio because either the vaccine did not provide them protection or OPV caused vaccine associated paralytic poliomyelitis (VAPP) these children are entitled to a suitable compensation.

Children are administered OPV soon after birth and many more doses are administered at different time under national polio eradication program. Vaccine coverage for children upto age of five years has not been $100 \%$. The likely effect of un-vaccinated and vaccinated children on polio eradication is presented below:

\section{Un-vaccinated child}

1. May not be infected by wild poliovirus.

2. Infected by wild poliovirus:

a. Develop paralytic disease and spread wild polioviruses to others. About 1 percent of infected children develop paralytic disease.

b. Develop immunity following infection.

\section{Vaccinated child}

1. Develop immunity and act as a barrier in spread of wild poliovirus in the community demonstrating herd protection by OPV [1].

2. Develop immunity and spread vaccine polioviruses in the community i.e. vaccinate close contacts by secondary spread

*Corresponding author: Yash Paul, Consultant Pediatrician, Maharaja Agrasen Hospital, Vidhyadhar Nagar, Jaipur 302023, India, E-mail: dryashpaul2003@yahoo.com

Received January 11, 2013; Accepted January 29, 2013; Published January 31, 2013

Citation: Paul Y (2013) Compassion and Compensation for Polio Cases. J Vaccines Vaccin 4: 170. doi:10.4172/2157-7560.1000170

Copyright: (c) 2013 Paul Y. This is an open-access article distributed under the terms of the Creative Commons Attribution License, which permits unrestricted use, distribution, and reproduction in any medium, provided the original author and source are credited. 
of vaccine polioviruses, and provide herd immunity. Now it is known that this benefit occurs on negligible scale only because of low infectivity of vaccine viruses and their small number in fecal matter, about 100 vaccine viruses are found in $1 \mathrm{gm}$ of stool [2].

3. Develop paralytic poliomyelitis due to mutant neurovirulent vaccine viruses called recipient vaccine associated paralytic poliomyelitis (VAPP).

4. Spread mutant neurovirulent vaccine viruses which may cause paralytic poliomyelitis in close contact called contact VAPP.

5. Spread vaccine derived polio viruses (VDPVs) in the community where mutant vaccine viruses recombine with community and cause paralytic disease in other susceptibles.

Thus, every un-vaccinated child may not develop polio and on the other hand many children have developed polio after taking many doses of polio vaccine as can be seen in table 1 .

\section{Informed Consent in the Polio Program} for ethical medicine [3]. The children who are administered OPV under polio eradication program are below legal age to give consent, but parents were not informed about the possible risks from OPV which had been known for a long time. In a publication entitled 'Together we make India polio free' produced jointly by the Indian Academy of Pediatrics and UNICEF following a workshop held in New Delhi on May 20-21, 2000 on page 14 under Issues and Concerns it was stated: (i) Public discussion of VAPP may cause serious damage to credibility of the polio eradication strategy (ii) In the present scheme VAPP is discarded as non-polio, although they are the unwanted product of polio programme, (iii) Epidemiological, clinical and laboratory investigation of VAPP is not carried out. Thus not only information regarding possible harm to a child by OPV was being held back, but facts regarding the occurrence of VAPP were being suppressed.

This author was a participant in that workshop, which was attended by experts from WHO, UNICEF, Government of India and IAP. Such a view might be justified on the ground that this polio eradication program would benefit the whole world. However, if it is indeed to be accepted that the benefits of polio eradication outweigh the withholding of information about the risks of harm, then, at the very least, an adequate compensation scheme needs to be in place for those that are harmed as a result of the program [4].

In any mass public health program some participants may not derive benefits due to some reasons, but harm should not occur to any participant. Given the benefits of OPV, polio cases because of OPV (VAPP) or because of vaccine failure where children developed paralytic polio despite taking adequate number of OPV doses may be viewed as an acceptable 'cost' of the program [5].

Question may be asked: should the cases of those children who developed paralytic polio during this campaign be considered as non-polio enteroviruses. Such VDPVs can circulate in the

Informed consent is held by many to be a necessary requirement

'acceptable cost'? Large number of polio cases in itself is not the only reason to make it unacceptable cost. Following are the additional reasons which make it unacceptable.

1. Only those children with residual paralysis after 60 days of onset of paralysis are considered either as confirmed polio cases if their stool samples were found positive for wild polio viruses or VAPP cases if their stool samples were positive for vaccine polio viruses. In those cases where stool samples could not be collected, or were not transported to the assigned laboratories in proper condition and thus may fail to isolate wild polioviruses or vaccine polio viruses are labelled as non-polio cases.

2. Now luckily polio cases by wild polio cases are not occurring after January 13, 2011, polio cases caused by OPV will continue to occur, but are not being labelled as polio cases. In 2011 there were 54 polio compatible cases and 7 cases by VDPVs. In year 2012 there were 10 polio compatible cases and 1 case by VDPVs. Number of VAPP cases are not being disclosed. Though not virologically confirmed polio cases, but nevertheless all these compatible polio cases and cases caused by VDPVs are paralytic polio cases, still we claim that no polio case has occurred in India after January 13, 2011.

3. It was known that OPV can cause VAPP, but during pulse polio immunization program people were told that OPV is absolutely safe, which was not the truth.

4. People were also told through media that OPV is highly effective, although children were developing polio despite taking large number of polio cases as can be seen in the following table 1 .

5. OPV administration has not been declared compulsory. People or the parents of the children have a right even to refuse a vaccine which has been declared compulsory, but, they have to give reasons for refusal and would be responsible for any harm occurred to them or their family members because of nonvaccination.

All national health programs should be implemented by persuation and not by coercion. But, OPV was being forced to all children upto age of five years. A news item published in Hindustan Times, New Delhi edition dated August 14, 2007 on page 9 under caption 'Refuse polio drops, lose power and ration cards' stated that "sub-divisional magistrate Raghuvir Yadav ordered immediate disconnection of power supply to the house of one Hafiz in Manechha village as well as cancellation of his ration card. Hafiz had refused to let his children be immunized on August 10. The same punishment was meted out to Ayub of Sabarhad. Other people were warned of similar action".

On September 3, 2007 the author had written to the Secretary, National Human Rights Commission, New Delhi in this regard. Assistant Registrar (Law) of the National Human Rights Commission through Letter Reference Case No. 27870/24/39/07-08/OC addressed to the Secretary, Department of Health \& Family Welfare, Govt. of Uttar Pradesh, Lucknow with a copy to the author stated: "The complaint dated 03.09.2007 received from Dr. Yash Paul in respect of Hafiz

\begin{tabular}{|c|c|c|c|c|c|c|c|c|c|c|c|c|}
\hline OPV Doses & 1998 & 1999 & 2000 & 2001 & 2002 & 2003 & 2004 & 2005 & 2006 & 2007 & 2008 & 2009 \\
\hline 0 Dose & $15 \%$ & $14 \%$ & $14 \%$ & $9 \%$ & $16 \%$ & $14 \%$ & $4 \%$ & $0 \%$ & $3 \%$ & $1 \%$ & $1 \%$ & $1 \%$ \\
\hline 1 - 3 doses & $47 \%$ & $45 \%$ & $28 \%$ & $31 \%$ & $41 \%$ & $35 \%$ & $11 \%$ & $11 \%$ & $10 \%$ & $3 \%$ & $3 \%$ & $4 \%$ \\
\hline 4 - 7 doses & $32 \%$ & $34 \%$ & $35 \%$ & $41 \%$ & $33 \%$ & $34 \%$ & $41 \%$ & $44 \%$ & $22 \%$ & $12 \%$ & $18 \%$ & $18 \%$ \\
\hline$>7$ dose & $7 \%$ & $8 \%$ & $23 \%$ & $18 \%$ & $11 \%$ & $17 \%$ & $44 \%$ & $45 \%$ & $65 \%$ & $85 \%$ & $78 \%$ & $77 \%$ \\
\hline
\end{tabular}

Table 1: Number of OPV doses received by polio cases, 1998-2009. 


\begin{tabular}{|l|l|l|l|l|l|l|l|l|l|l|l|l|l|l|l|l|l|l|l|l|l|l|l|l|}
\hline Polio Cases iwn India & 1998 & 1999 & 2000 & 2001 & 2002 & 2003 & 2004 & 2005 & 2006 & 2007 & 2008 & 2009 & 2010 & 2011 & 2012 \\
\hline & 1934 & 1126 & 265 & 268 & 1600 & 225 & 136 & 66 & 676 & 874 & 559 & 741 & 42 & 1 & 0 \\
\hline
\end{tabular}

Table 2: Number of polio cases in India from 1998-2012 as on 27th December, 2012

and others was placed before the Commission on 08.10.2007". Upon perusing the complaint, the Commission directed as follows:

"The complaint is transmitted to the concerned authority for such action as deemed appropriate. Accordingly I am forwarding a copy of the complaint to you for its disposal at your end". Despite many reminders, no further communication was received.

The author had raised the issue with the Medico-legal Group of Indian Academy of Pediatr [6]. The experts had stated: All national health programs need to have persuasive tone and should never have coercive tenure. Coercion may arrogate with fundamental right enshrined in Article 21 of Constitution of India [7].

\section{Who Should Pay Money for Compensation?}

There are three components in mass public health projects: (i) policy making, (ii) implementation of the project and (iii) funding of the project. In case project succeeds credit goes to policy makers and those who have implemented the program and donors are thanked for the financial support. But, if the project fails, i.e. desired results are not achieved, some unforeseen harm occurs or more harm than anticipated occurs to the participants, the whole project needs to be re-assessed, necessary changes be made or the project dropped. But, in case of polio eradication program no such steps were taken till 2005 when monovalent polio vaccines were introduced. But new vaccine failed to reduce the polio incidence, which actually increased and later bivalent polio vaccine was introduced and polio incidence reduced as can be seen in table 2 . Thus, remedial steps were taken very late, but no steps are being taken to stop occurrence of VAPP. Thus responsibility to pay compensation lies with policy makers unless it is proved that the implementation of the program was at fault.

\section{Who are Eligible for Compensation?}

Polio cases can be divided in five groups: (i) who did not receive polio vaccine, (ii) who received adequate doses of OPV but failed to develop adequate antibodies to protect against disease i.e., cases of vaccine failure, (iii) who developed VAPP following administration of OPV, (iv) close contacts of vaccine who developed VAPP due to secondary spread of mutant neurovirulent vaccine viruses, and (v) who developed paralysis because of VDPVs.

As no proper documents are provided to the parents regarding OPV administration under polio eradication program so it should be presumed that all children had participated in the program. National polio eradication program was started on October 2, 1995 so that every polio case that was born on 2nd October 1995 and later or any other date fixed by consensus is eligible for compensation. If given option some children might not have been administered OPV and escaped VAPP or had been administered IPV and thus not developed polio either as vaccine failure case or VAPP.

Policy makers were fully aware of the fact that some children may not be protected by OPV and could develop paralytic polio and some children may develop polio because of OPV called VAPP. The official data showed that number of such polio cases occurring in India was much higher than expected. The projected number of VAPP cases in a year for India was 60 [8], according to official data there were 181 VAPP cases in 1999 [9], but according to the estimates made by the author, about 300 VAPP cases occur every year in India [10].

Contributions made by different agencies and donors should be acknowledged and active role played by millions of volunteers who had worked tirelessly for polio eradication should be highlighted. The children who have been afflicted with permanent disability should not be treated as a price to be paid in larger interest, but should be given appropriate compensation on ethical and legal grounds. Every person who develops harm due to failure of a drug or adverse drug reaction is entitled for damages and compensation, then why children who have been harmed by OPV should be excluded on the ground that it was part of a global program.

Some high level national committee or commission should be constituted to decide on the amount of compensation depending on degree of residual paralysis. Medical boards consisting of pediatrician, orthopedic surgeon and neurologist should be constituted at least at every district head quarter to diagnose and evaluate degree of residual paralysis.

\section{References}

1. Paul Y (2004) Herd immunity and herd protection. Vaccine 22: 301-302.

2. Chen RT, Hausinger S, Dajani AS, Hanfling M, Baughman AL, et al. (1996) Seroprevalence of antibody against poliovirus in inner-city preschool children. Implications for vaccination policy in the United States. JAMA 275: 1639-1645

3. Dawson A (2003) Informed consent: Should we really insist upon it? New Rev Bioeth 1: 59-71.

4. Paul Y, Dawson A (2005) Some ethical issues arising from polio eradication programmes in India. Bioethics 19: 393-406.

5. Dawson A, Paul Y (2006) Mass public health programmes and the obligations of sponsoring and participating organisations. J Med Ethics 32: 580-583.

6. Paul Y (2009) Punishment for Refusing OPV. Indian Pediatr 46: 540-541.

7. Tiwari SK, Baldwa M (2001) Medical negligence. Indian Pediatr 38: 488-495.

8. John TJ (1996) Can we eradicate poliomyelitis. Frontiers in Pediatrics, Jaypee Brothers, New Delhi, India.

9. Kohler KA, Banerjee K, Gary Hlady W, Andrus JK, Sutter RW (2002) Vaccineassociated paralytic poliomyelitis in India during 1999: decreased risk despite massive use of oral polio vaccine. Bull World Health Organ 80: 210-216.

10. Paul Y (2004) Need for re-appraisal of acute flaccid paralysis (AFP) case classification. Vaccine 22: 3829-3830 